\title{
LOW ENERGY DEMONSTRATION ACCELERATOR (LEDA) BEAM INSTRUMENTATION: RFQ-ACCELERATED BEAM RESULTS *
}

\author{
J. D. Gilpatrick, D. Barr, J. Power, W.C. Sellyey, R. Shurter, M. Stettler, LANL, Los Alamos, NM \\ J. Kamperschroer, D. Martinez, General Atomics, Los Alamos, NM \\ J. O’Hara, AlliedSignal Inc., Los Alamos, NM
}

\begin{abstract}
Beam diagnostic instrumentation is being developed for the LEDA, a $6.7-\mathrm{MeV}, 100-\mathrm{mA}-\mathrm{cw}$ proton accelerator, presently being commissioned at the Los Alamos National Laboratory (LANL). This instrumentation will be the basis for much of the Accelerator Production of Tritium and the Spallation Neutron Source linac. Located in the LEDA injector and the high energy beam transport (HEBT) this initial instrumentation suite's purpose is to verify the RFQ pulsed and cw operation. The instrumentation include a series of DC, pulsed- and bunched-beam current measurements from which RFQ beam-transmission efficiency will be determined. Ionization-chamber beam loss measurements are mounted above the HEBT and provide input signals to a fast equipment protection system. Central beam phase and energy measurements provide RFQ longitudinal performance information. Beam position measurements provide information to properly center the beam within the HEBT beam pipe. Finally, two types of transverse profile measurements including a slow wire scanner and a video fluorescence monitor provide beam width and projection information in the LEDA HEBT. This paper will discuss these measurements developed for LEDA and summarize how they performed during RFQ verification experiments.
\end{abstract}

\section{INTRODUCTION}

The LEDA RFQ has been installed and, as of March 16, 1999, has accelerated beam [1]. Beam instruments located in a simple HEBT were developed, fabricated, and installed to initially verify 100-mA RFQ operation [2]. To minimize the risk to the HEBT beamline equipment from beam impingement damage, the RFQ has been initially operated with a beam repetition rate, pulse length, and peak current to $10 \mathrm{~Hz}, 3 \mathrm{~ms}$, and $40 \mathrm{~mA}$, respectively. Therefore, the beam instrumentation has been required to operate during both pulsed and cw beam operation.

\section{INSTRUMENTATION LOCATION}

Most of the diagnostics instrumentation is located in the HEBT [3], pictured in Figure 1. The DC and pulsed beam-current measurements are placed near the RFQ's entrance and exit to determine the RFQ total beam transmission. A third set of beam current measurements is placed near the entrance to the beamstop to determine the HEBT beam transmission.

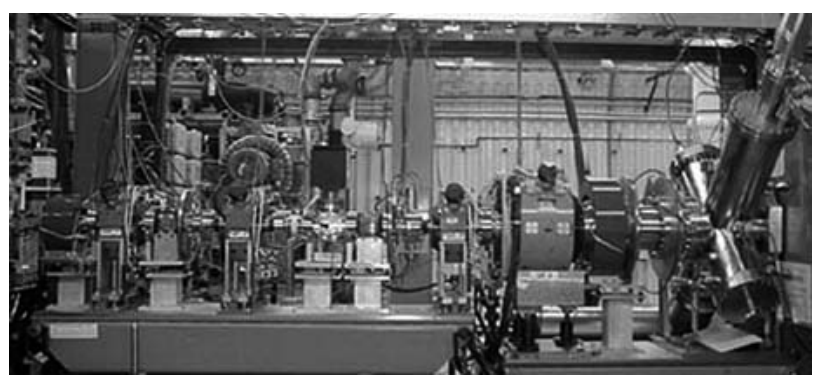

Figure 1: This picture shows the assembled HEBT including magnetic transport elements (i.e., four quadrupole and two steering magnets) and beam instrumentation.

Ionization chamber loss measurements are placed at the RFQ exit, at the middle of the HEBT, and at the entrance to the beamstop. These chambers, located at the beamline's elevation but approximately 1 meter from the beamline, provide fast beamline-equipment protection and additional beam transport tuning information.

Three capacitive probes detect the beam's central phase and energy. Beam energy is calculated by detecting the flight time for a bunch to travel between two probes. The first two probes, located approximately $0.5 \mathrm{~m}$ from the RFQ exit, are separated by approximately $0.1 \mathrm{~m}$ and provide approximate beam energy information. The first and third probes are separated by approximately $1 \mathrm{~m}$ and provide the more accurate energy information. The central phase is acquired by measuring the phase between an RFQ field sample probe and the first capacitive probe.

Five beam position monitors (BPM) are located throughout the HEBT. These micro-stripline BPMs are at locations sensitive to beam position variations due to changes in beam angle at the RFQ exit and the two HEBT steering magnets. BPMs \#4 and \#5 are located after the final quadrupole magnet for monitoring the beam's final trajectory to the beamstop. In addition, each BPMs' fourelectrode signals are summed to provide bunched beam current information [4]. Since the RFQ's 6.7-MeV beam is captured within the $350-\mathrm{MHz}$ RF bucket, the bunched beam current amplitude provides a reliable measure of the relative accelerated-beam current.

\footnotetext{
* Work supported by the US Department of Energy.
} 
A slow wire scanner is positioned just in front of the beamstop to assess the expanded beam's size at the end of the beamstop and to measure the emittance of the beam using a "quadrupole scan" technique [5]. In the near future, a background gas-fluorescence beam-profile measurement will be placed at this same longitudinal HEBT location to measure cw beam profiles.

\section{MEASUREMENT DISCUSSION}

\subsection{Pulsed Beam Current}

The pulsed beam current measurements use a single toroidal transformer core with two sets of secondary windings: one set for beam current detection and another set for an in situ calibration [6]. The current detectionwinding signals are initially processed using a simple analog feedback amplifier to increase the innate transformer L/R time constant, therefore, reducing the droop of the transformer signal. The low-droop signals from two transformers (e.g., RFQ entrance and exit) are digitized and are manipulated in digital signal processing (DSP) circuitry to produce a 200-kHz-bandwidth differential-current measurement. This digital difference signal is then integrated for approximately 2 millisecond and compared to a fast protection lost-charge value. If the lost charge is greater than this value, the fast protection system is initiated and discontinues beam operation.

\subsection{Central Beam Phase and Energy}

Since these electronics processors are still being fabricated, the phase and energy measurements were initially performed with only the installed capacitive probes, phased stabilized RF cables, a down converter, and an oscilloscope. The down converter transfers the 350$\mathrm{MHz}$ phase information from the capacitive probes to 2 $\mathrm{MHz}$ where phase may be more accurately converted to time. Because the distance between probes is known, the energy can then be accurately calculated.

\subsection{Beam Loss (Ionization Chambers)}

Beam loss measurements use ionization chambers to detect the interaction between lost protons and beamline components [7]. Electron signal currents created and collected from the result of gamma-radiation ionizing the $\mathrm{N}_{2}$ chamber gas. These electron signal currents are then amplified and integrated and are compared to a fast protection lost-charge value. If the integrator signals are greater than this value, the fast protection system is initiated and discontinues beam operation. These three chamber integrated signals are also monitored to provide beam tuning information.

\subsection{Beam Position and Bunched Beam Current}

Since the BPM electronics processors are also still being fabricated, we used only the analog front end (AFE) circuitry from the DSP-based BPM electronic processors.
The AFE circuitry filters, amplifies, and detects BPM electrode signals in such a way as to provide a logarithmic amplitude of the $350-\mathrm{MHz}$ electrode signals. For these initial RFQ beam measurements, the individual signal amplitudes were quantified using an oscilloscope and subtracted to provide an approximate measure of beam position using the log ratio technique. The AFE circuitry also acquires bunched beam current by summing the fourelectrode processed signals.

\subsection{Beam Profiles}

The slow wire scanner consists of an actuator that is mounted with a 45-degree angle with respect to the vertical and horizontal axes. SiC 0.1-mm-diameter fibers are mounted on the actuator "fork". Two fibers are used as bias to optimize the secondary electron coefficient of the fiber and the remaining fiber senses the beam. The wires traverse from one tine of the fork to the other vertically, then turn 90 degrees and traverse back. With such a system, horizontal and vertical beam profiles may be acquired in a single sweep. The beam charge-density information is acquired by measuring the beam-induced secondary electrons emitted from the fiber. Beam charge density data and fiber position are both acquired and controlled with LabVIEW ${ }^{\mathrm{TM}}$ software [8].

\section{INITIAL RESULTS}

The pulsed current measurements at the RFQ exit and entrance to the HEBT beamstop show good agreement with theory and are consistent with each other and their in situ calibration hardware. Their $200-\mathrm{kHz}$ bandwidth precision and accuracy are measured to be $<0.1 \mathrm{~mA}$ and $<0.25 \mathrm{~mA}$, respectively. However, under certain beam conditions, the RFQ-entrance current measurement appears to have an error during beam operation that is not present during the in situ calibration procedure. This error results in a higher than expected beam transmission through the RFQ. This error is presently being investigated and will be corrected. Figure 2 displays a partial LabVIEW ${ }^{\mathrm{TM}}$ screen that reports the pulsed beam current data with data.

Preliminary loss measurement observations performed during wire-scanner profile measurements indicate that the ionization chambers beam loss precision is approximately $0.2 \mathrm{~mA}$.

Beam energy was measured with both a short and long drift distances between capacitive probes. For the longdrift-distance energy measurements, the initial measurement precision and accuracy are $<1 \mathrm{keV}$ and $<10$ $\mathrm{keV}$, respectively. Figure 3 displays both energy measurements and the results from simulating the transport and the acceleration of the beam through the RFQ. The two measurements appear to be consistent and agree well with the RFQ simulations.

The wire scanner measurements also performed as expected. With amplifier gains set so that the peak of the 
charge distribution is below their saturation limits, the ratio of fiber signals acquired at the distribution peak and tails was measured to be $>500: 1$. The combination of this large signal-to-noise ratio and the small $0.1-\mathrm{mm}$ diameter fiber widths will allow this profile measurement to acquire valid profile data beyond $3 \mathrm{rms}$ widths.

The final two beam position measurements have been shown to be consistent with each other, steering magnet currents, and the nearby wire scanner profile measurement.
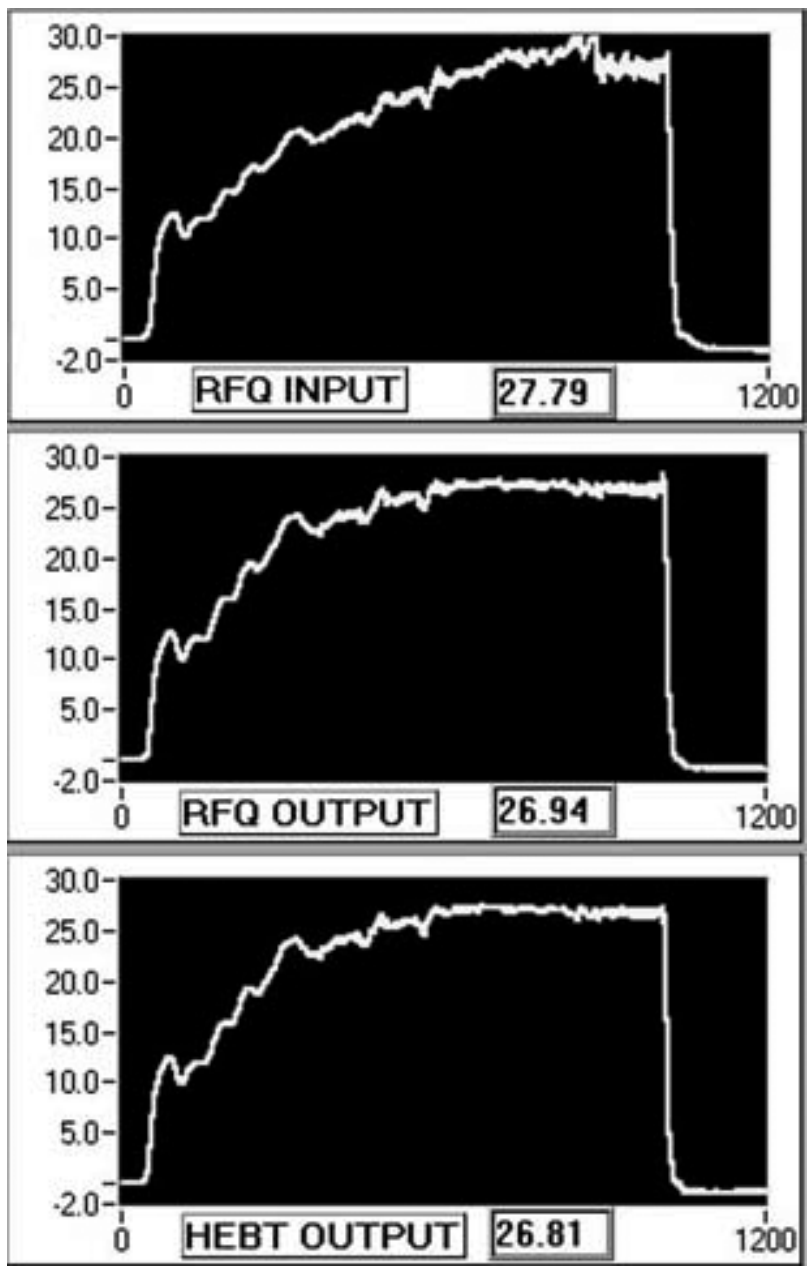

Figure 2: This picture shows the pulsed beam current at the entrance and exit of the RFQ and at the entrance of the HEBT beamstop. The abscissa and ordinate units are $\mu \mathrm{s}$ and $\mathrm{mA}$, respectively. The values in the three smaller boxes under each waveform are beam currents typically averaged during the final $300 \mu$ s of the macropulse.

\section{CONCLUSIONS}

During the past 9 days of initial LEDA RFQ operation, most of the beam diagnostic measurements have been preliminarily tested with beam. With a few exceptions, most appear to be operating as expected. With additional beam commissioning time, the beam instrumentation will be fully commissioned and ready for further RFQ studies.

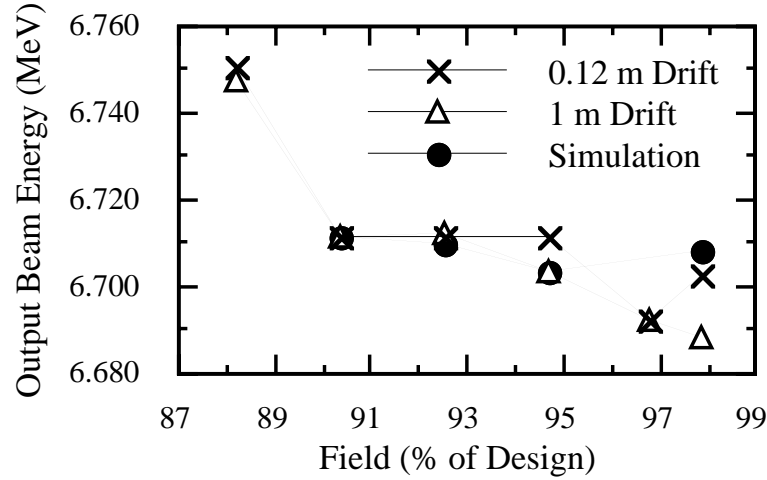

Figure 3: This graph illustrates the RFQ measured outputbeam energy as a function of the percent of the RFQ designed field. Within measurement error, the two energy measurements are consistent and compare favorably with the simulation.

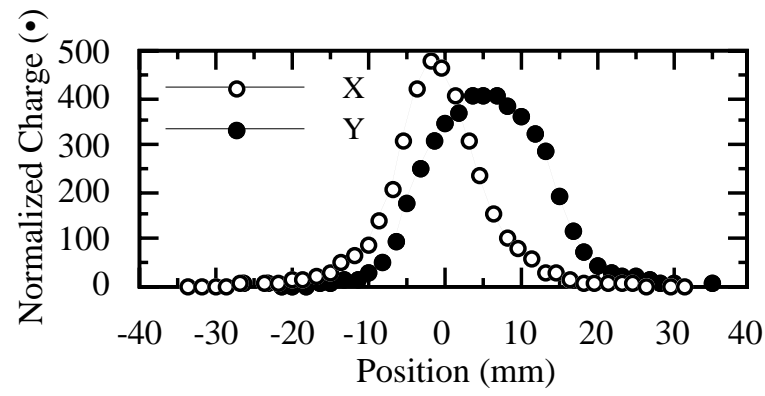

Figure 4: The above graph shows typical data acquired from the wire scanners located approximately $2.8 \mathrm{~m}$ from the LEDA RFQ exit. The horizontal beam location and rms width and the vertical beam location and rms width are $-0.9,6.2,5.9$, and $6.8 \mathrm{~mm}$, respectively.

\section{REFERENCES}

[1] J. D. Schneider, et al., "Operation of the Low-Energy Demonstration Accelerator: the Proton Injector for APT," these proceedings.

[2] J. D. Gilpatrick, et al., "LEDA and APT Beam Diagnostics Instrumentation," PAC '97, Vancouver, BC, Canada, June, 1997.

[3] W. Lysenko, et al., "High Energy Beam Transport Beamline for LEDA," XIX International Linear Accelerator Conference, August 23-28, Chicago, IL, 1998.

[4] J. D. Gilpatrick, et al., "LEDA \& APT Beam Position Measurement System: Design and Initial Tests," XIX International Linear Accelerator Conference, August 23-28, Chicago, IL, 1998.

[5] W. P. Lysenko, private communication (1998).

[6] J. Power, et al., "Beam Current Measurements for LEDA," these proceedings.

[7] J. D. Gilpatrick, et al., "Experience with the Ground Test Accelerator Beam Measurement Instrumentation," in AIP Conference Proceedings 319, (Santa Fe, NM, August, 1993), p. 154.

[8] LabVIEW $^{\mathrm{TM}}$ is commercial software sold by National Instruments, Inc. 